\title{
Polit-ökonomische Aspekte des Berichts zur Lage der Verbraucher:innen
}

\author{
Der Sachverständigenrat für Verbraucherfragen hat erstmals ein breit angelegtes Gutachten \\ zur Lage der Verbraucher:innen in Deutschland vorgelegt. Gert G. Wagner, Mitglied im \\ Sachverständigenrat für Verbraucherfragen, stellt aus seiner persönlichen Sicht ausgewählte \\ Analysen, Ergebnisse und Empfehlungen vor. Über das Gutachten hinausgehend wird auch \\ auf relevante Ereignisse hingewiesen, die nach Abschluss des Gutachtens stattfanden: die \\ Vorlage des EU-Verordnungsvorschlags „Artificial Intelligence Act“, das Klimaschutz-Urteil des \\ Bundesverfassungsgerichts und der Gesetzentwurf zum autonomen Fahren.
}

Mit dem im April 2021 vorgelegten Gutachten zur Lage der Verbraucher:innen in Deutschland erfüllt der seit 2014 beim Bundesministerium der Justiz und für Verbraucherfragen angesiedelte Sachverständigenrat für Verbraucherfragen (SVRV) erstmals seinen aus dem Einrichtungserlass resultierenden Auftrag, in regelmäßigem Abstand ein Gutachten vorzulegen (SVRV, 2021, 19). Ziel des Gutachtens ist es, aus Sicht der Verbraucher:innen auf die deutsche Gesellschaft, insbesondere die Volkswirtschaft, zu schauen und wesentliche Probleme und Herausforderungen darzustellen sowie ausgewählte Handlungsempfehlungen für die Verbraucherpolitik auszusprechen. ${ }^{1}$

Insgesamt bewertet der SVRV (2021, 6 und 412) die Lage der Verbraucher:innen „im Allgemeinen nicht als grundsätzlich besorgniserregend“. Das ist nicht falsch, aber der Autor dieser Zeilen hält dies für eine übertrieben zurückhaltende Einschätzung. Ich persönlich bewerte die Lage der Verbraucher:innen als überwiegend gut - was aber auch bedeutet, dass Gruppen, die in einer schlechteren Lage sind, umso mehr eine politische Herausforderung sind. Man wird diesen Gruppen nur helfen können, wenn man in der Mitte der Gesellschaft, der es gut geht, Mehrheiten generiert. Und das gelingt nicht, wenn man der Mehrheit

(C) Der/die Autor:in(nen) 2021. Open Access: Dieser Artikel wird unter der Creative Commons Namensnennung 4.0 International Lizenz veröffentlicht (creativecommons.org/licenses/by/4.0/deed.de).

Open Access wird durch die ZBW - Leibniz-Informationszentrum Wirtschaft gefördert.

* Der Autor dankt den weiteren Mitgliedern und den Mitarbeitenden des SVR für Verbraucherfragen. Er gibt hier seine persönliche Sicht auf das gemeinsam verfasste Gutachten wieder. Nur längere wörtliche Zitate sind kenntlich gemacht.

1 Ob und wie sich die Pandemie auf die Verbraucher:innen mittel- und längerfristig auswirkt, war zum Zeitpunkt der Finalisierung des Gutachtens (Ende Januar 2021) noch nicht absehbar. So werden nur punktuelle Befunde referiert, aber es gibt kein Corona-Kapitel. einreden will, es ginge ihr gar nicht so gut, wie sie sich in Deutschland seit Jahren fühlt (Priem et al., 2021). Hieran hat auch die Corona-Pandemie nicht grundsätzlich und effektiv etwas geändert (Liebig und Kühne 2021; SVRV, 2021, 54). Um es deutlich zu sagen: Berechtigte Beschwerden und Klagen der Haushalte sowie der Verbraucherverbände sind mit der Einschätzung einer guten Lage der großen Mehrheit der Verbraucher:innen voll und ganz kompatibel. Viele öffentliche und veröffentlichte Diskussionen sowie juristische Klagen sind nicht der Beweis einer schlechten Lage, sondern ein Beleg für einen funktionierenden demokratischen Prozess und einen funktionierenden Rechtsstaat.

\section{Strukturprobleme}

Dass neue Technologien und Einsatzbereiche, die wegen ihres großen Nutzens rasch weite Verbreitung finden, Probleme verursachen, ist nicht überraschend, sondern - wie der SVRV (2021, 6 und 412) betont - zu erwarten. Wie das z.B. gegenwärtig bei der Digitalisierung der Fall ist, mit Defiziten in der Infrastruktur, großen sozialen Unterschieden bei der Nutzung und aus Verbrauchersicht unbefriedigenden oder gar betrügerischen Angeboten. Dabei ist es kein grundsätzliches Problem, dass die für den Verbraucherschutz angemessenen Regulierungen erst nach und nach auf Basis von Versuch und Irrtum etabliert werden - das war bei jeder neuen Technologie so und wird auch in Zukunft so sein.

Prof. Dr. Dr. h. c. Gert G. Wagner ist Senior Fellow am Deutschen Institut für Wirtschaftsforschung (DIW Berlin), Max Planck Fellow am MPI für Bildungsforschung in Berlin und Mitglied des Sachverständigenrats für Verbraucherfragen. 
Dass aber im Bereich der Ernährung Fehlernährung Probleme verursacht, ist hingegen - wie der SVRV feststellt - ein strukturelles Problem. „Strukturell problematisch ist auch in den Bereichen Wohnen und Mobilität ein Zielkonflikt, der sich für viele Menschen daraus ergibt, dass sie ruhig und bezahlbar wohnen möchten - dieser Wunsch sich allerdings insbesondere in Großstädten nicht leicht realisieren lässt und viele Menschen deswegen im Umland oder auf dem Land wohnen. Aus dem dadurch bedingten räumlichen Auseinanderdriften von Arbeit, Wohnen und Freizeit ergeben sich Mobilitätszwänge und möglicherweise eine dauerhaft abgesenkte Lebenszufriedenheit" (SVRV, 2021, 6). Hier reichen bessere Informationen, auf die seit Jahren die systematische Verbraucherpolitik setzt, nicht aus. Verbraucherpolitik ist mehr als Informationsverbesserung. Ein Beispiel sind auch die zunehmenden Schäden, die das Klima verursacht: Hier schlägt der SVRV im Kapitel zum Wohnen eine Versicherungspflicht gegen Elementarschäden vor (SVRV, 2021, 51 f.; ausführlich Groß et al., 2019). Auch für das Wohnen reicht es nicht aus, nur bessere Informationen und Klagemöglichkeiten zu Verfügung zu stellen: „Der Staat sollte seiner Verantwortung gerecht werden und die Rahmenbedingungen dafür schaffen, dass auch Wohnungen im unteren und mittleren Preisniveau weiter gebaut werden. Jenseits der Verbraucherpolitik im engeren Sinne ist und bleibt nach Einschätzung des SVRV sozialer Wohnungsbau auf Dauer erforderlich, um ein passendes und bezahlbares Wohnangebot sicherzustellen“ (SVRV, 2021, 91).

\section{Nachhaltiger Lebensstil}

Die vielleicht wichtigste Aussage im Gutachten ist, dass „nachhaltiger Konsum ein für das Überleben der Menschheit notwendiges Ziel“" ist (SVRV, 2021, 9 und 223). Diese Prämisse ist offensichtlich mit dem inzwischen vorliegenden Urteil zum Klimaschutz des Bundesverfassungsgerichts (2021) kompatibel. Um mehr Nachhaltigkeit des Konsums zu erreichen, reicht eine bessere Verbraucherschutzpolitik offensichtlich nicht aus, also „lediglich bessere Informationen für Konsumenten“, etwa über die Herkunftsländer von Gewürzen, Gemüse und Früchten, sondern „es ist ein nachhaltigkeitsorientierter Ordnungsrahmen notwendig und entsprechende staatliche Strukturmaßnahmen," wodurch schließlich Verbraucher:innen „selbstbestimmte Entscheidungen im Einklang mit Nachhaltigkeit treffen können“ (SVRV, 2021, 9).

Zu einem Nachhaltigkeits-Ordnungsrahmen bzw. zu ordnungspolitischen Maßnahmen für mehr nachhaltigen Konsum sagt der SVRV nichts weiter, denn wenn auch eine sozial ausgewogene Ausgestaltung angestrebt wird, wozu sich der SVRV abstrakt bekennt, fällt es doch jedem pluralistisch besetzten Beratungsgremium schwer, sich einerseits auf Empfehlungen von Verboten bestimmter
Produkte sowie verhaltenslenkende Steuern und Abgaben zu einigen und andererseits auf sozial kompensierende Maßnahmen, etwa bei der Besteuerung oder gezielten Transfers. ${ }^{2}$ Immerhin kann die Empfehlung des SVRV, das Wissen über Nachhaltigkeit zu stärken (NachhaltigkeitsLiteracy), indirekt auch ordnungspolitische Maßnahmen und gezielte Steuern und Abgaben nach sich ziehen: Wenn besser informierte Wähler:innen in den Parlamenten für Mehrheiten sorgen, die Strukturmaßnahmen nachhaltig implementieren. Der SVRV hält ausdrücklich fest, dass - selbst bei guter Nachhaltigkeits-Literacy (SVRV, 2021, 235 f.) - souveräne Konsumentscheidungen zugunsten der ökologischen Nachhaltigkeit oft nicht einfach sind, da nicht überall nachhaltige Produkte angeboten werden und es oftmals schwer fällt, Informationen darüber zu finden, ob ein Produkt nachhaltig hergestellt wurde und wie die Lieferketten aussahen (SVRV, 2021, 268f.).

Verbraucherschutzpolitik beruht traditionell auf bestimmten Ge- und Verboten, so speziell im Ernährungsbereich. In den vergangenen Jahrzehnten ist die Verbesserung der Informationen, die über Konsumgüter und Wohnungen zur Verfügung gestellt werden müssen, immer mehr in den Vordergrund gerückt. Etwa im Bereich Ernährung werden die Inhaltsstoffe von Lebensmitteln und deren Nährwert differenziert dargestellt sowie oft deren Regionalität und Herkunft mitgeteilt. Auf der anderen Seite zeigt eine Bevölkerungsbefragung des SVRV, dass mehr als $70 \%$ der befragten Verbraucher:innen es eher als schwierig einschätzen, Informationen darüber zu finden, ob ein Produkt nachhaltig ist. Das Fehlen verständlicher Nachhaltigkeitskennzeichnungen wird von $31 \%$ als ein Hinderungsgrund für nachhaltiges Konsumieren angegeben. Daher empfiehlt der SVRV die Verbraucherinformationen hinsichtlich nachhaltiger Konsumoptionen verständlicher und übersichtlicher zu gestalten und das Angebot an nachhaltigen Produkten zu fördern (SVRV, 2021, 268 ff.).

\section{Verbraucherpolitik im traditionellen Sinne}

Der oder die eigenverantwortlich handelnde Verbraucher:in ist als normatives Leitmotiv ein zentraler Bestandteil einer marktwirtschaftlichen Wirtschaftsordnung. Maßnahmen zur Verbesserung der Informationslage und der Entscheidungskompetenz sind aufgrund der mit der Digitialisierung verbundenen neuen Möglichkeiten nach wie vor zentral für den Verbraucherschutz. Ein kompetenter Umgang mit dem Internet ist die Voraussetzung dafür, dass Verbraucher:innen die potenziellen Vorteile digitaler Märkte - besserer Zugang zu Informationen, eine größere Auswahl, potenziell niedrigere Preise und mehr Innovation bei Produkten und Dienst-

2 Der SVRV besteht aus sieben wissenschaftlichen Mitgliedern verschiedener Disziplinen und zwei Praxisvertretern (für Angebot und Nachfrage). 
leistungen - für sich realisieren und Souveränität in der digitalen Welt erlangen können. Dabei ist es nicht überraschend, dass die für das SVRV-Gutachten durchgeführten Studien zeigen, dass die digitalen Verbraucherkompetenzen in Deutschland noch große Lücken aufweisen und vor allem sozial ungleich verteilt sind (SVRV, 2021, 399). So wissen z. B. nur ca. $25 \%$ der Verbraucher:innen sicher über ihr Rückgaberecht bei Online-Käufen Bescheid. Die Verbraucherpolitik ist daher gefordert, differenzierte Maßnahmen zu ergreifen, um die digitalen Verbraucherkompetenzen zu erhöhen. Dies ist leichter gesagt als getan, da die größten Lücken bei bildungsfernen und älteren Menschen zu finden sind und Erwachsenenbildung schwierig ist. In der digitalen Welt muss Verbraucherpolitik deshalb mehr sein als die Verbesserung der Verbraucherinformation: Informationsverpflichtungen für Anbietende müssen um Maßnahmen des rechtlichen Verbraucherschutzes ergänzt werden. Freilich sind Informationspflichten ein zeitintensives und kostspieliges Instrument, das in der Praxis wenig oder keine Wirkung zeigt, da die Informationsflut die Menschen überfordert bzw. auf kein Interesse stößt (SVRV, 2021, Kapitel E). So zeigt eine Befragung, dass nur etwa die Hälfte der Nutzer:innen von Vergleichs- und Vermittlungsplattformen überhaupt Interesse an Transparenzinformationen hat und diese kaum genutzt werden (SVRV, 2021, 10).

Mehr Informationen führen nicht unbedingt zu mehr Informiertheit. Daher empfiehlt der SVRV (2021, 11 und 400) nicht noch mehr Regulierung, welche die Informationsflut noch vergrößert, „sondern eine bessere und gezieltere Regulierung der Verbraucherinformationen“, also eine "Lichtung des Regelungsdickichts“, eine Reduzierung auf das Wesentliche. Entsprechend sollte die Verbraucherpolitik einen gesetzlichen Rahmen für Gütesiegel schaffen. Das ist natürlich leichter gesagt als getan, denn es ist alles andere als offenkundig, was das Wesentliche ist. Deswegen empfiehlt der SVRV unter dem Label „Verbraucherinformatik“ (SVRV, 2021, 393) mehr und gezielte Forschung. Gemeint ist damit Forschung zu Risikokommunikation. Zum Beispiel sollten auf Basis von Abbildungen oder grafischen Symbolen zügig „Nichttextliche-Informationsmodelle" entwickelt und empirisch daraufhin überprüft werden, ob sie die effektive Nutzung von Informationen durch die Verbraucher:innen, etwa bei Plattformanbietern, steigern könnten (vgl. Kasten 1).

\section{Informationen und bessere Bildung über Ernährung}

Ernährung ist ein zentraler und komplexer Konsumbereich, der gegenwärtig etwa $15 \%$ des durchschnittlichen Konsumbudgets in Anspruch nimmt. Ernährung ist ein wesentlicher Bestandteil des Alltags und dient auch der Identitäts- und Lebensstilbildung (SVRV, 2021, Kapitel C.III). Allein aus diesen Anforderungen entstehen bereits Probleme (z.B. durch knappe Einkommen und den Wunsch einen sozial anerkannten Lebensstil zu pflegen), hinzu kommt eine unübersichtliche Zahl von Waren. Die Untersuchungen des SVRV zeigen, dass aus Verbrauchersicht nicht nur die Kosten der Ernährung wichtig sind, sondern auch die Inhaltsstoffe und Nährwertkennzeichnung, Gütesiegel, Regionalität und Herkunft von Lebensmitteln sowie - für kleine Verbrauchergruppen - der Fleischkonsum. Dabei sind Verbraucher:innen mit Migrationshintergrund aufgrund religiöser Anforderungen an Lebensmittel mit weiteren besonderen Problemlagen konfrontiert.

Viele Menschen zeigen sich hinsichtlich Lebensmitteln stark verunsichert und erwarten eine stärkere und vor allem übersichtlichere staatliche Regulierung und Kontrolle. Daher empfiehlt der SVRV, durch bessere und transparente Lebensmittelkontrollen Vertrauen zu schaffen (2021, 205 f.). Der Gütesiegel-Dschungel sollte gelichtet und Kennzeichnungen bezüglich der regionalen Herkunft, des Tierwohls und der Nachhaltigkeit von Lebensmitteln klarer geregelt werden. Neben staatlichen Gütesiegeln sollten alternative Siegel, etwa von Unternehmen, „nur zulässig sein, wenn ihre Güte bzw. tatsächliche Aussagekraft für alle Verbrauchergruppen nachweisbar über die Mindeststandards staatlicher Siegel hinausgeht." Das muss effektiv kontrolliert werden, dafür dürften übergeordnete Kontrollbehörden und -einrichtungen besser geeignet sein als Städte und Kommunen.

Ein schwieriges Kapitel ist der Fleischkonsum (SVRV, 2021, 208ff.). Der SVRV geht davon aus, dass Anreize für einen reduzierten Fleischkonsum zwar gesundheitlich und ökologisch sinnvoll sind, aber dass auch die meisten weiterhin Fleisch essen wollen und eine stark einschränkende Regulierung z. B. durch eine gezielte Besteuerung als unerwünschten schweren Eingriff in die Privatsphäre empfinden würden. Statt Fleischkonsum stark zu reglementieren und zu verteuern, empfiehlt der SVRV daher Maßnahmen, die an der grundsätzlichen Bereitschaft vieler ansetzen, ihren Fleischkonsum zu reduzieren. Der SVRV befürwortet gezielte Informationen, um besser über die Folgen des Verzehrs von Fleisch und anderen Tierprodukten und über die Vorteile einer Umstellung auf eine stärker pflanzlich basierte Ernährung zu informieren. Und die „Bundesregierung sollte (endlich) ein verpflichtendes, staatliches Tierwohllabel einführen und sich darüber hinaus weiterhin für eine einheitliche, verpflichtende Kennzeichnung auf EU-Ebene einsetzen. Die Verbraucher:innen sollten durch die Kennzeichnung transparent über die Tierwohlleistungen eines konkreten Produktes informiert werden. Ein Tierwohllabel sollte mehrstufig sein, wobei sich die Kriterien des Labels nicht daran orientieren sollten, was für Landwirte einfach und kostengünstig umsetzbar ist, sondern die Kriterien sollten wesentliche Steigerungen des Tierwohls belohnen. Aus diesem Grund sollten die Vergabekriterien für höhere Stufen des Labels auch im Laufe der Zeit schrittweise angehoben werden“ (SVRV, 2021, Empfehlung 24). 
Kasten 1

Ausgewählte Empfehlungen des SVRV zu Datenerhebungen und Forschungsorganisation

\section{Datenerhebungen}

Hinsichtlich der systematischen Förderung nachhaltiger Konsummuster empfiehlt der SVRV (2021, 421) der Bundesregierung, ein Monitoring zu etablieren, das die Entwicklungen und Veränderungen im Bewusstsein, der Kompetenz und Gelegenheitswahrnehmung hinsichtlich nachhaltigen Konsums erfasst. Die für das Lage-Gutachten durchgeführte Bevölkerungsbefragung könnte ein Ausgangspunkt sein. „Im Zuge des Monitorings sollten auch die Messinstrumente und die sozioökonomische Differenzierung in der Methode und Auswertung validiert und weiterentwickelt werden und zum Zwecke der evidenzbasierten Maßnahmenevaluation eingesetzt werden."

Weiterhin empfiehlt der SVRV (2021, 8, 47, 88 und 412) „den Wohngeld- und Mietenbericht der Bundesregierung um eine Bestandsaufnahme der Situation von (selbstnutzenden) Eigentümerinnen und Eigentümern sowie Menschen in Gemeinschaftsunterkünften und wohnungslosen Menschen zu ergänzen“. Folgerichtig wäre es, diesen Bericht als „Wohnbericht“ zu bezeichnen. Da praktisch alle empirischen Erhebungen, so auch der für den SVRV durchgeführte Survey, die Lebenssituation wohnungsloser und in Heimen und Gemeinschaftsunterkünften lebender Menschen nicht berücksichtigen, weist der SVRV besonders auf die Notwendigkeit einer systematischen deutschlandweiten statistischen Erfassung hin. Das Wohnungslosenberichterstattungsgesetz (WoBerichtsG) wird ausdrücklich begrüßt, das erstmals für 2022 eine Erhebung einer Statistik über wohnungslose Menschen vorsieht.

Für den Bereich der Mobilität empfiehlt der SVRV $(2021,415)$ „die Entwicklung von empirischen Indikatoren zur Evaluierung der Grundversorgung im Bedarfsfeld Mobilität“.

\section{Organisation der Verbraucherforschung}

Hinsichtlich der Verbraucherforschung in Deutschland stellt der SVRV (2021, 11 und 426) resümierend fest: „In der Verbraucherpolitik werden an vielen Stellen Informationen und Erkenntnisse benötigt, die derzeit nicht vorliegen (...). Deswegen sei abschließend darauf hingewiesen, dass die Verbraucherforschung in Deutschland darunter leidet, dass es keine grundfinanzierten Forschungseinrichtungen gibt, die schwerpunktmäßig Verbraucherforschung betreiben und mit anderen Forschungseinrichtungen, die sich mit Fragen z. B. der Ernährung, der Wohn- und Infrastruktur, der Mobilität, der Daseinsvorsorge, der Digitalisierung, der Nachhaltigkeit oder der Verbraucherinformation befassen, permanent zusammenarbeiten können." Deswegen empfiehlt der SVRV „die Einrichtung von zwei wissenschaftsgetragenen außeruniversitären Zentren für Verbraucherforschung, die bundesseitig vom für Verbraucherfragen zuständigen Bundesressort finanziert werden“. Bei dieser Empfehlung sind die Begriffe sorgfältig gewählt: Es werden nicht zwei völlig neue Institute gefordert, sondern „lediglich“ zwei permanente Zentren, die in bestehenden außeruniversitären Instituten aber auch an Universitäten eingerichtet werden könnten. Hier sei auch darauf hingewiesen, dass bewusst zwei Zentren empfohlen werden, um - wie der SVRV (2021, 11 und 426) betont - „ein Mindestmaß an wissenschaftlichem Wettbewerb zwischen den Zentren [zu] schaffen und so zur Qualitätssicherung der Forschung bei[zu]tragen. Zugleich wäre gewährleistet, dass das breite Themen- und Methodenspektrum der Verbraucherforschung angemessen abgedeckt wird“.

Hinzu kommt aber noch ein weiterer Aspekt: Um Konkurrenz zu schaffen, dürfen zwei solcher Zentren nicht so spezialisiert sein, dass sie faktisch keine Konkurrenten sind. Sondern beide müssen die grundlegenden Fragen der Verbraucherforschung bearbeiten, um Konkurrenz zu gewährleisten. Dadurch würde es zumindest gelegentlich auch zu öffentlichem Streit der beiden Einrichtungen kommen und dieser würde nach Überzeugung des Autors das Feld der Verbraucherforschung keineswegs schwächen. Öffentliche Auseinandersetzungen tragen zum Agenda Setting bei - sie unterstreichen also die gesellschaftliche Bedeutung eines Politik- und Forschungsfeldes. Ein Beispiel für relevante Forschung nennt der SVRV (2021, 206 und 401) ausdrücklich, nämlich die wissenschaftlichen Grundlagen für „wissenschaftlich fundierter Kriterien zwingende Mindeststandards für die Qualität von Gütesiegeln, Mindestanforderungen für die Überwachung der Einhaltung dieser Kriterien nach dem Vorbild der in der Datenschutzgrundverordnung (Art. 40 ff.) niedergelegten Ko-Regulierung, die Einbindung in die nationale Akkreditierungsstelle der Bundesrepublik Deutschland sowie das Verfahren der staatlichen Anerkennung und Überwachung von Siegeln und Zertifikaten sowie die Kenntlichmachung staatlich anerkannter Siegel und Zertifikate". 
Ein Forschungsfeld nennt der SVRV $(2021,390)$ ausdrücklich als prioritär: die optimale Regulierung von algorithmischen Entscheidungen und Künstlicher Intelligenz (IZ). Es ist nicht nur aufgrund der rasanten Entwicklung dieser Felder wichtig, sondern auch deswegen, weil es noch keine geordnete Forschungslandschaft gibt, die die zu schaffenden Regulierungsbehörden unterstützen kann. Die Dringlichkeit entsprechender Forschung wird durch die von der EU-Kommission vorgelegten Vorschläge zu Verordnungen über digitale Dienste und Märkte (Digital Services Act und Digital Markets Act) sowie Künstliche Intelligenz (Artificial Intelligence Act) unterstrichen. Zur Überwachung von Algorithmen hat sich der SVRV in seinem Gutachten „Verbrauchergerechtes Scoring“ (2018) bereits differenziert geäußert. Im Nachgang zu diesem Gutachten haben Gerberding und Wagner (2019) gezeigt, dass bereichsspezifische gesetzliche Regelungen notwendig sind. Abstrakte Regelungen (wie z.B. in der von der EU-Kommission vorgelegten KI-Verordnung) werden nicht ausreichen. Selbst komplizierte Algorithmen (sogenannte selbstlernende Systeme) sind überwachbar, wenn die Gesetzgebung Schnittstellen erzwingt, durch welche die Wirkungsweise von Algorithmen anhand von Testdaten, die man in den Algorithmus gibt, studiert wird (Gigerenzer et al., 2018). Für solche Schnittstellen ist es nicht notwendig, dass der oder die Eigentümer:in des Algorithmus den Code, d. h. das geistige Eigentum, offenlegt. In diesem Zusammenhang ist es hochinteressant, dass im Gesetzentwurf der Bundesregierung zum autonomen Fahren ausdrücklich der Zugang der Forschung zu den beim autonomen Fahren produzierten Daten geregelt wird (Bundesregierung 2021, 11). Mithilfe dieser Daten kann die KI geprüft werden, die autonome Fahrzeuge steuert. Die Daten sollen laut Gesetzentwurf in anonymisierter Form vom Kraftfahrt-Bundesamt bereitgestellt werden, das darauf auch vorbereitet ist, da es ein Forschungsdatenzentrum als Schnittstelle zur unabhängigen Forschung betreibt, das vom Rat für Sozial- und Wirtschaftsdaten (www.ratswd.de) zertifiziert wurde (https://www.kba.de/DE/Statistik/Forschungsdatenzentrum/Ansprechpartner/ansprechpartner_node.html).

Da unser Ernährungsverhalten ganz maßgeblich in der frühen Kindheit geprägt wird, ist eine verbesserte Verbraucherbildung insbesondere in Schulen und Kindertagesstätten sinnvoll. Wichtig ist die Empfehlung des SVRV (2021, Empfehlung 22), „die Ernährungs- und Verbraucherbildung in einem schon bestehenden AnkerSchulfach oder einem neu einzurichtenden Schulfach bundesweit verpflichtend zu verankern, die Aus- und Weiterbildung von Lehrkräften für Verbraucherbildung an Universitäten aufzubauen und zu fördern; die sachlichen und personellen Rahmenbedingungen für diesen Bildungsbereich deutlich auszubauen und die Verbraucherkompetenzen bei Schüler:innen regelmäßig zu evaluieren." Dabei könnte das Mittagessen in Ganztagsschulen für die Verbraucherbildung genutzt werden.

\section{Bessere öffentliche Mobilitätsangebote und eine bessere Verteilung des öffentlichen Raums}

Im Bereich der Mobilität werden die Grenzen traditioneller Verbraucherschutzpolitik besonders deutlich - bessere Informationen helfen nur begrenzt weiter, um die Lage der Verbraucher:innen im Bereich Mobilität zu verbessern, vielmehr sind Infrastruktur- und Baupolitik gefragt (SVRV, Kapitel C.II). Wie in allen Industriegesellschaften unterliegen auch in Deutschland die Menschen einem hohen Mobilitätsdruck: Viele Erwerbstätige müssen im Alltag zwischen Wohn- und Arbeitsort pendeln und fast alle müssen zwischen Einkaufsorten, Ämtern, Versorgungsinstitutionen und Freizeitstätten Wege zurücklegen. Wer Sorgeverpflichtungen hat, muss oft zusätzlich Wege zu Kindertagesstät- ten, Schulen, Alters- und Pflegeheimen etc. bewältigen. Ebenso wie für die Ernährung gibt ein Durchschnittshaushalt ungefähr $15 \%$ seines Budgets für Mobilität aus; für Verbraucher:innen mit niedrigem Einkommen ist die finanzielle Belastung wesentlich (SVRV 2021, 125 ff.). Dabei sind in den vergangenen Jahren vor allem die Preise im öffentlichen Verkehr stärker gestiegen als die Einkommen. Der SVRV empfiehlt daher die Prüfung alternativer Finanzierungsmodelle, welche die finanzielle Belastung der Nutzer:innen des öffentlichen Verkehrs senken können (SVRV, 2021, Empfehlung 11). Wesentlich ist jedoch, dass gleichzeitig eine Angebots- und Qualitätssteigerung erfolgt, denn auch ein kostenloser ÖPNV wird nur genutzt, wenn er attraktiv ist und die Mobilitätsbedürfnisse bedient.

In der Tat werden über alle sozialen Gruppen und Regionen hinweg Versorgungslücken und Belastungen bei öffentlichen Verkehrsmitteln gesehen. Beklagt werden mangelnde Angebote, fehlende Zuverlässigkeit und hohe Kosten. Der Fahrradverkehr sollte aus Sicht vieler sicherer und attraktiver werden. Vor diesem Hintergrund sollte die Verbraucherpolitik nach Ansicht des SVRV $(2021,415)$ erreichen, „dass Versorgungslücken im öffentlichen Verkehr beseitigt werden. Dabei gilt es, sowohl den Zugang zum öffentlichen Verkehr zu verbessern als auch dessen Attraktivität (Zuverlässigkeit, Komfort, Sicherheit) zu erhöhen. In den Ballungszentren muss der Fokus auf einer Neuverteilung des öffentlichen Raums zugunsten des Umweltverbunds (zu Fuß gehen, Rad fahren, öffentlicher Verkehr) liegen und Multimodalität für Verbraucher:innen erleichtert werden." 
Weiterhin empfiehlt der SVRV (2021, Empfehlung 13), das politische Ziel einer autogerechten Stadt zu überwinden. „Vor allem das Ziel der ökologischen Nachhaltigkeit kann nur durch eine Verlagerung vom Auto auf den Langsamverkehr (zu Fuß gehen, Fahrradfahren) und den öffentlichen Verkehr (Zug, S-Bahn, U-Bahn, Tram, Bus) erreicht werden." Hierzu sind vor allem infrastrukturelle Maßnahmen im Bereich des Städtebaus, der Stadt- und der Verkehrsplanung erforderlich. Der SVRV weist darauf hin, dass es für die Entwicklung einer menschengerechten Stadt zentral ist, eine gerechte Verteilung der Flächen des öffentlichen Raums unter der Berücksichtigung der Bedürfnisse aller Verkehrsteilnehmenden zu realisieren. „Vor diesem Hintergrund empfiehlt der SVRV die Institutionalisierung eines nationalen Dialogs über die notwendigen Voraussetzungen für die Entwicklung und Umsetzung alternativer nachhaltiger und integrativer Mobilitätskonzepte, insbesondere über die Einführung eines Leitbildes, nach dem Fahrräder und öffentliche Verkehrsmittel in der Verkehrsplanung auf allen Ebenen vorrangig zu berücksichtigen sind."

\section{Verbessertes Wohnen mehr als Verbraucherschutz}

Ein Durchschnittshaushalt gibt etwa $35 \%$ seines Budgets für Wohnen aus. Im Durchschnitt geht dieser große Ausgabenblock auch mit einer hohen Zufriedenheit einher: Seit Jahren liegt die Wohnzufriedenheit über der allgemeinen Lebenszufriedenheit (SVRV, 2021, Kapitel C.I). Eine im Durchschnitt hohe Wohnzufriedenheit ist ohne Zweifel auch eine Folge des differenzierten Mietrechts, das Mieter:innen gut schützt. Probleme treten freilich vorgelagert auf, nämlich in Form von Informationsproblemen bei der Suche nach einer Wohnung oder einem Haus, und beim Vertragsabschluss. ${ }^{3}$ Trotz aller Negativmeldungen über Wohnprobleme zeigen empirische Analysen, dass die Zufriedenheit mit dem Wohnen in den letzten Jahrzehnten kontinuierlich zugenommen hat. Dabei haben sich 30 Jahre nach der Wende in Ost- und Westdeutschland die Werte auf hohem Niveau nahezu angeglichen: Sie liegen im Durchschnitt bei etwa 8 von 10 möglichen Zufriedenheitspunkten. Diese Werte übertreffen die Bewertung der allgemeinen Lebenszufriedenheit, die im Moment im Durchschnitt bei etwa 7 von 10 Punkten liegt. Hier ist die Lücke zwischen Ost und West immer noch deutlich erkennbar. Und anders als die allgemeine Lebenszufriedenheit wurde die Wohnzufriedenheit bislang auch kaum durch die Folgen der Pandemie getrübt, wie die Erhebungen COMPASS und SOEP-CoV zeigen. Dieser überraschende Befund dürfte daran liegen, dass Homeoffice eher von Personen in Anspruch genommen wird, die genug Platz haben

3 Auf Phänomene wie die Vermietung von Wohnraum an Touristen (Duso et al., 2020a,b) und aktuelle Entwicklungen wie die beabsichtigte Fusion großer Wohnungsunternehmen (Michelsen, 2021) und den Berliner Mietendeckel geht das Gutachten wegen seines Redaktionsschlusses, nicht ein (Kholodilin und Michelsen, 2020; Hahn et al., 2021; Michelsen 2021a). und im Schnitt etwas zufriedener mit ihrer Wohnung sind als andere. Kinder, die laut Studien besonders unter der Isolation durch wiederholte Lockdowns leiden, werden nicht zur Wohnzufriedenheit befragt. Für Erwachsene zeigt sich, dass Wohnzufriedenheit klar vom Alter und Einkommen abhängt: je älter und wohlhabender, desto zufriedener. ${ }^{4}$

Es zeigt sich auch (SVRV, 2021, 79), dass hohe Wohnkosten oft kein Problem sind, da diese meist nur von denjenigen gezahlt werden, die über ein überdurchschnittliches Einkommen verfügen. Deswegen ist in Großstädten die Unzufriedenheit mit den Wohnkosten keineswegs überdurchschnittlich, da dort auch die Einkommen höher sind als in anderen Städten und Gemeinden. Problematisch sind vielmehr „normale“ bis moderat hohe Wohnkosten, die auf niedrige Einkommen treffen. Das größte Problem ist in bestimmten Regionen und Städten das Finden einer geeigneten Wohnung. Hat man erst eine Wohnung gefunden, greift das deutsche Mietrecht und die Zufriedenheit ist meist hoch. Aber: Das Fünftel der Mieter:innen, das in der SVRVErhebung (September 2020) seine Wohnkosten als „unangemessen“ bezeichnet, gibt nur eine durchschnittliche Wohnzufriedenheit von 6,1 an, im Vergleich zu 7,8 bei Mietenden, die ihre Wohnkosten als angemessen bezeichnen und 8,9 bei Eigentümer:innen. Die konkreten Bedürfnisse bezüglich "guten Wohnens" sind je nach Lebenslage sehr unterschiedlich (SVRV, 2021, 64 ff.). Ruhe ist bei allen Gruppen wichtig, aber Mietende und Eigentümer:innen unterscheiden sich deutlich: Für Mieter:innen spielt Bezahlbarkeit die größte Rolle, für Eigentümer:innen Ruhe. Und hohe Wohnkosten sind keineswegs pauschal mit Unzufriedenheit verbunden, da hohe Kosten oft mit einem größeren Platzbedarf und höherem Einkommen zusammenhängen. Bei unterdurchschnittlichen Haushaltseinkommen dominiert der Wunsch nach Bezahlbarkeit, bei überdurchschnittlichen Einkommen der Wunsch nach Ruhe und Platz (vgl. auch Abbildung 1).

Die überwiegend hohe Zufriedenheit mit dem Wohnen bedeutet aus Sicht des Autors, dass umso mehr für diejenigen getan werden sollte, die in schlechten und beengten Wohnverhältnissen leben - das gilt speziell für jüngere Menschen und für Haushalte mit Kindern. Die Notwendigkeit zum Handeln besteht allerdings über die klassische Verbraucherpolitik hinaus, wie sich an den repräsentativ befragten Personen zeigt, die als wichtigste Merkmale „guten Wohnens" die „Ruhe“ und „Bezahlbarkeit“ nennen. Zwar lässt sich Ruhe im Kleinen durch den Einbau von Schallschutzfenstern erreichen (SVRV, 2021, 92 f. und 414 f.). Im Großen sind jedoch Infrastrukturmaßnahmen erforderlich, die bis in

4 Der Autor dankt Dr. Christian Groß von der Geschäftsstelle des SVRV für Unterstützung bei der Analyse der aktuellen Daten, die über das Gutachten hinausgehen, sowie Dr. Nico A. Siegel und Christian Spinner für die Überlassung von Mikro-Daten des COMPASS-Online-Surveys, auf dem die hier vorgelegten aktuellen empirischen Ergebnisse beruhen. 
Abbildung 1

Persönliche Relevanz der Facetten guten Wohnens bei Haushalten mit deutlich unterdurchschnittlichem Haushaltsnettoeinkommen, September 2020
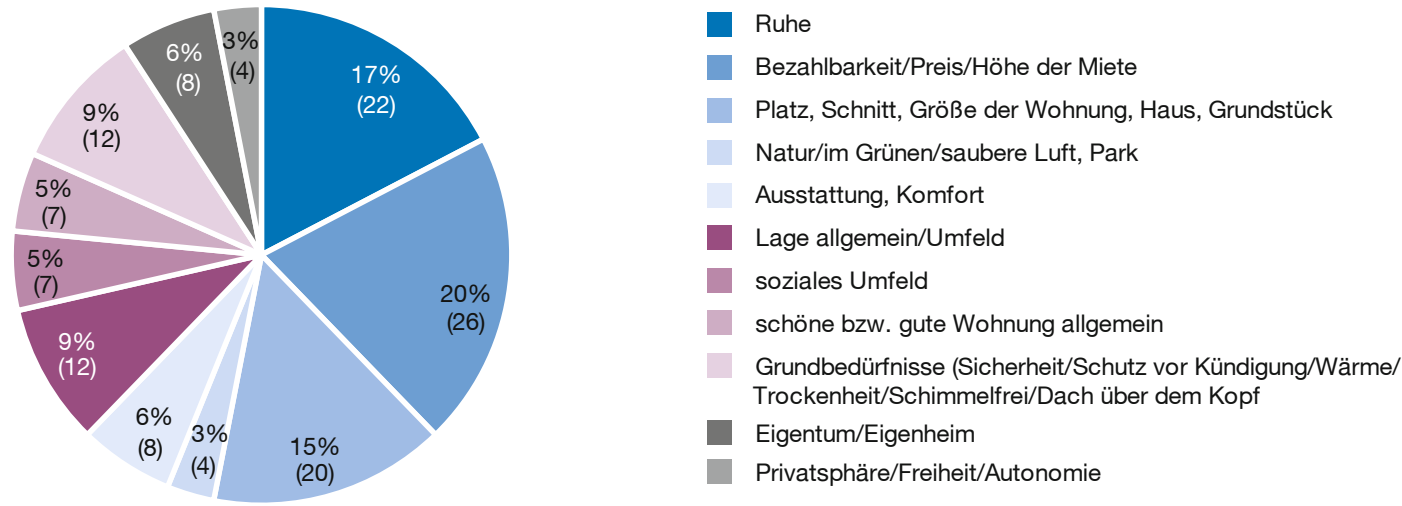

Anmerkungen: Gewichtete Werte, Anteile (gerundet in \%, absolute Zahlen in Klammern) der erstgenannten Antworten auf die offen gestellte Frage „Das Thema dieser Befragung ist „Gut Wohnen in Deutschland“. Hier interessiert uns zunächst ganz allgemein in lhren eigenen Worten, was „Gutes Wohnen“ für Sie ganz persönlich ausmacht. Was ist Ihnen dabei wichtig?“, von elf Kategorien (Elf Facetten guten Wohnens) auf Basis einer Codierung der transkribierten Freitexte (eigene Berechnung und Darstellung auf Basis der SVRV-Erhebung, n = 519; die Antwortkategorien „Sonstiges“ und „weiß nicht“ sind nicht abgebildet). Unterdurchschnittliche Haushaltnettoeinkommen bis 2.500 Euro; der Durchschnitt betrug 2018 rund 3.660 Euro.

Quelle: Eigene Auswertung einer für den SVRV im September 2020 durchgeführten INFAS-Telefonerhebung.

die Verkehrspolitik (Verkehrsberuhigung) hineinreichen. Und das Kriterium der Bezahlbarkeit reicht offensichtlich in den Bereich der Wohnungsbaupolitik hinein - immer dann, wenn insbesondere in Großstädten zu wenig Wohnraum zur Verfügung steht. Jenseits der Verbraucherpolitik bleibt sozialer Wohnungsbau auf Dauer erforderlich, um ein passendes und bezahlbares Wohnangebot zu gewährleisten. Der Staat sollte seiner Verantwortung gerecht werden, die Rahmenbedingungen dafür zu schaffen, dass auch Wohnungen im unteren und mittleren Preissegment weiter gebaut werden.

Bedacht werden sollte auch, dass hohe Mietkostenbelastungen im unteren Einkommensbereich nicht zwangsläufig durch überhöhte Mieten zustande kommen müssen, sondern auch unangemessen niedrige Löhne können daran schuld sein. Man denke etwa an die in der Corona-Krise erkannte Unterbezahlung von Pflegekräften. Kurzfristig würde ein zielgerecht verbessertes Wohngeld wahrscheinlich effektiver helfen als ein Mietendeckel, der naturgemäß auch Leute begünstigt, die große Wohnungen problemlos bezahlen können. Mittel- und langfristig hilft sozialer Wohnungsbau am effektivsten, um die Wohnkostenbelastung in Grenzen zu halten - es helfen aber auch höhere Löhne in schlechtbezahlten Berufen.

\section{Ausblick}

Das Gutachten zur Lage der Verbraucher:innen macht deutlich, dass moderne Verbraucherpolitik mehr ist als Verbraucherschutzpolitik. Das Einkommen wird nicht nur für Konsum verwendet, sondern auch für Wohnen, Mobilität und
z.B. Altersvorsorge. Verbraucherpolitik ist letztlich Gesellschaftspolitik. Damit liegt - über das Gutachten hinaus - die Frage nahe, wo die Verbraucherpolitik ministeriell angesiedelt sein sollte. Ich habe mich seit meiner Berufung in den SVRV immer wieder gefragt, ob das für Justiz zuständige Bundesministerium wirklich ein guter Ort ist. Vor dem Wechsel in das Justizministerium war die Verbraucherpolitik im für die Landwirtschaft zuständigen Bundesministerium angesiedelt, also in einem Ministerium, das mit der Ernährung für einen großen und wichtigen Konsumbereich zuständig ist.

Landesministerien zeigen eine bunte Vielfalt der Ressortzuordnung der Verbraucher(schutz)politik. Sie wird kombiniert mit Ernährung, Europa, Gesundheit, Integration, Justiz, Landwirtschaft, ländlichem Raum, Naturschutz, Sozialem und Umwelt. Für alle Kombinationen gibt es nachvollziehbare Gründe, aber letztlich ist jede Zuordnung zu einem Ministerium mit Spezialaufgaben willkürlich. Auf Bundesebene ist die Zuordnung zum Justizministerium insofern sehr plausibel, als sich dieses Ministerium in alle Gesetzgebungsverfahren einmischt und insofern ein im Grundsatz guter Ort für eine Querschnittsaufgabe wie die Verbraucherpolitik ist. Dass es in einem Justizministerium Nicht-Juristen eher schwer haben, ist allerdings ein nennenswerter Nachteil - er wird freilich umso kleiner, je länger die Verbraucherpolitik im Bundesministerium der Justiz ressortiert.

Eine Merkwürdigkeit könnte bei der nächsten Regierungsbildung mit einem Federstrich geändert werden - wobei die damit verbundene Symbolik aber wichtig wäre. Im Gegensatz zum Sachverständigenrat für Verbraucherfragen 
ist das Bundesministerium der Justiz für Verbraucherschutz zuständig. Moderne Verbraucherpolitik geht aber weit über traditionellen Verbraucherschutz hinaus. Der Widerspruch zwischen der Benennung des Ministeriums und der Benennung des Sachverständigenrats ist wahrscheinlich in der Hektik der Koalitionsverhandlungen niemandem aufgefallen, oder es sollten keine schlafenden Hunde geweckt werden. Bei den kommenden Koalitionsverhandlungen könnte zumindest darüber geredet werden, ob die verschiedenen Bezeichnungen gewollt sind.

Abschließend sei ein Punkt angesprochen, der nicht nur für den SVRV relevant ist. Nämlich die Frage, wie die Bundesregierung mit Sachverständigenberichten und -empfehlungen umgeht. Man kann immer wieder beobachten, dass Expertenberichte und Empfehlungen von der Politik mehr oder weniger ignoriert werden. Dies mag in vielen Fällen oder vielleicht auch nur gelegentlich sachlich sinnvoll sein, da ein Bericht und Empfehlungen nicht besonders gewichtig sind. Aber gerade dann wäre eine explizite Reaktion der Politik für alle Beteiligten, auch die Öffentlichkeit, aufschlussreich; jedenfalls besser als Schweigen oder der formal freundliche Hinweis, dass man einen Bericht und seine Empfehlungen nun prüfen werde. Für eine bessere Governance des Umgangs mit wissenschaftlichen Ergebnissen könnte der Sachverständigenrat für die Beurteilung der gesamtwirtschaftlichen Entwicklung dienen, der auf einem Gesetz beruht, das auch festschreibt, dass die gesamte Bundesregierung zu dem Jahresgutachten folgenden Jahreswirtschaftsbericht Stellung nehmen muss. Diese Stellungnahme kann natürlich rein routinemäßig und formelhaft ausfallen. Aber zumindest gelegentlich entspinnt sich eine für Politik, Wissenschaft und Öffentlichkeit interessante und relevante Diskussion. Und genau das sollen Sachverständigenräte ja bewirken (Sager und Wagner, 2019; Wagner, 2013a, b).

\section{Literatur}

Bundesministerium für Verkehr und digitale Infrastruktur (2017), Bericht der Ethik-Kommission „Automatisiertes und vernetztes Fahren, https://www.bmvi.de/SharedDocs/DE/Publikationen/DG/berichtder-ethik-kommission.pdf?_blob=publicationFile (1. Juni 2021).

Bundesregierung (2021), Entwurf eines Gesetzes zur Änderung des StraBenverkehrsgesetzes und des Pflichtversicherungsgesetzes - Gesetz zum autonomen Fahren, Deutscher Bundestag, Drucksache 19/27439, 9. März 2021.

Bundesverfassungsgericht (2021), Beschluss des Ersten Senats vom 24 März 2021, 1 BvR 2656/18, Rn. 1-270, Karlsruhe:ECLI:DE:BVerfG:202 1:rs20210324.1bvr265618.

Duso, T., C. Michelsen, M. Schäfer und K. Tran (2020), Airbnb and Rents: Evidence from Berlin, DIW Discussion Paper, 1890.

Duso, T. , C. Michelsen, M. Schäfer und K. Tran (2021), Durch AirbnbVermietungen steigen in Berlin die Mieten, DIW Wochenbericht, 7, 95-102.

Gerberding, J. und G. G. Wagner (2019), Gesetzliche Qualitätssicherung für „Predictive Analytics“ durch digitale Algorithmen, Zeitschrift für Rechtspolitik, 52(4), 116-119.

Gigerenzer, G., F. G. Rebitschek und G. G. Wagner (2018), Eine vermessene Gesellschaft braucht Transparenz, Wirtschaftsdienst, 98(12), 860-866, https://www.wirtschaftsdienst.eu/inhalt/jahr/2018/heft/12/beitrag/eine-vermessene-gesellschaft-braucht-transparenz.html (1. Juni 2021).

Groß, C., G. G. Wagner und R. Schwarze (2019), Maßnahmen für eine zukunftsgerechte Naturgefahren-Absicherung, Policy Brief des SVRV, https://www.svr-verbraucherfragen.de/dokumente/massnahmen-fuereine-zukunftsgerechte-naturgefahren-absicherung/ (1. Juni 2021).

Hahn, A. M., K. Kholodilin und S. Waltl (2021), Die unmittelbaren Auswirkungen des Berliner Mietendeckels: Wohnungen günstiger, aber schwieriger zu finden, DIW Wochenbericht, 8, 117-124.

Kholodilin, K. und C. Michelsen (2020), Wohnungsmarkt in Deutschland: Trotz Krise steigende Immobilienpreise, Gefahr einer flächendeckenden Preisblase aber gering, DIW Wochenbericht, 37, 642-652.

Liebig, S. und S. Kühne (2021), Während der Corona-Pandemie sind die Menschen zunehmend unzufrieden mit der Freizeit, aber weiterhin zufrieden mit ihrem Schlaf, SOEP-CoV Spotlight, \#5, https://www.soepcov.de/Spotlight_5/.

Michelsen, C. (2021a), Regulierung bleibt sinnvoll, in: Wirtschaftsdienst, 101(5), 324

Michelsen, C. (2021b), Fusion von Vonovia und Deutsche Wohnen würde europäischen Immobilienriesen schaffen, https://www.diw.de/de/ diw_01.c.818576.de/fusion_von_vonovia_und_deutsche_wohnen_wuerde_europaeischen_immobilienriesen_schaffen.html (1. Juni 2021).

Priem, M., F. Kaiser und J. Schupp (2020), Zufriedener denn je - Lebensverhältnisse in Deutschland 30 Jahre nach dem Mauerfall, Informationsdienst Soziale Indikatoren, 64, 7-15.

Sager, K. und G. G. Wagner (2019), Wissenschaft unter Druck: Vertrauensverlust oder Zeichen gewachsener gesellschaftlicher Relevanz?, in W. Hinsch und D. Eggers (Hrsg.), Öffentliche Vernunft, 21-34.

SVR Gesundheit (Sachverständigenrat zur Begutachtung der Entwicklung im Gesundheitswesen) (2021), Digitalisierung für Gesundheit Ziele und Rahmenbedingungen eines dynamisch lernenden Gesundheitssystems - Gutachten 2021, https://www.svr-gesundheit.de/ fileadmin/Gutachten/Gutachten_2021/SVR_Gutachten_2021.pdf (1. Juni 2021).

SVRV (Sachverständigenrat für Verbraucherfragen) (2018), Verbrauchergerechtes Scoring, https://www.svr-verbraucherfragen.de (1. Juni 2021).

SVRV (Sachverständigenrat für Verbraucherfragen) (2021), Gutachten zur Lage der Verbraucherinnen und Verbraucher, https://www.svrverbraucherfragen.de, (1. Juni 2021).

Wagner, G. G. (2013a), BIPplus: Ein neue Wohlstands-Berichterstattung braucht mehr als nur bessere Statistiken, Universitas, 68(800), 56-63.

Wagner, G. G. (2013b), W3-Indikatoren sind ein handfestes Ergebnis der »Wohlstands-Enquete«, ifo Schnelldienst, 66(15), 7-11.

Title: Political-Economic Aspects of the Report on the Position of Consumers by the Advisory Council for Consumer Affairs (SVRV) Abstract: For the first time, the German Advisory Council for Consumer Affairs (SVRV) has published a comprehensive report on the position of consumers in Germany. The author, who is a member of the SVRV, presents selected analyses, results and recommendations. Additionally, relevant events that have taken place after the report was completed are also mentioned: the presentation of the proposed EU regulation "Artificial Intelligence Act", the climate protection ruling of the Federal Constitutional Court and the draft law on autonomous driving in Germany.

JEL Classification: A12, A29, D11, D12, D18, D31, D82, L66, O18, Q18, Q56, R41 